\title{
Torsional Strength in Fused Filament Fabrication 3D Printing
}

\author{
Nathan $\mathrm{Ng}^{1}$ and Kate Flowers ${ }^{1}$ \\ ${ }^{1}$ Santa Clara High School, Santa Clara, CA, USA
}

\section{ABSTRACT}

Among robotics enthusiasts, fused filament fabrication (FFF) 3D printing has exploded in popularity. The ability to manufacture custom parts allows for a greater degree of freedom during the design phase. For example, mechanisms like the drivetrain, a hotbed of torsion forces, can be easily compressed to make room for additional functionality. However, such specific components are often unavailable for purchase. Therefore, the ability to manufacture custom torsionally resistant parts in an inexpensive manner is essential. The objective of this study is to determine the FFF 3D printer settings that contribute significantly to a part's torsional strength. This will be accomplished by altering either infill percentage or wall line count during printing while keeping all other settings constant among 30 individual test parts. Each part will be tested in a custom testing machine that applies a 9:1 gear reduction to simulate the maximum output torque of most hobbyist drivetrains. By measuring the amount of time each test part lasts before snapping, the setting granting the greatest increase in torsional strength can be determined.

\section{Introduction}

Across the professional and hobbyist landscapes, 3D printing has exploded in popularity. One survey of over 1300 professionals across 12 different industries found that $51 \%$ apply 3D printing processes during the production stage. A majority of these experts additionally voiced their belief that 3D printing will play a major role in both manufacturing and business in the near future ("The State of 3D," 2019). In addition to eliminating many of the manufacturing constraints contemporary technologies like CNC machining suffer from, 3D printing allows for the rapid production of different iterations of prototypes in a matter of hours.

A similar expansion has taken place in the hobbyist market where 3D printing has become increasingly more accessible as manufacturing costs lower. A popular example is the well-received Creality Ender 3, an FFF 3D printer that has consistently reached sub-\$200 prices ("Ender 3 3D Printer," 2020). FFF 3D printers in particular have received the greatest attention for identical reasons: FFF 3D printers and filament are more inexpensive than alternative 3D printing processes.

Another key factor in the rise of 3D printing technologies on both fronts was the expiration of key 3D printing design patents. "Beginning in 2007 and peaking in 2014 however, several major 3D printing technology patents expired, allowing the low-cost personal/desktop 3D printer market to significantly expand, and directly leading to the 500K unit milestone [in 2015]" ("Global 3D printing market," 2019). Without patents enforcing a monopoly on the technology, a plethora of companies were able to produce the machines on both a wider scale and at a lower cost, again increasing the overall popularity of 3D printers.

However, 3D printing still lacks innovation in its optimization, especially in the hobbyist market, where individual designers lack the time and resources to actively research and develop their profiles. Both the radio-controlled car and robotics community fall under this category. Additionally, both heavily rely on FFF 3D printing for inexpensive parts requiring torsional strength like pulleys, sprockets, and axle hubs. As such, a need arises for a series of FFF 3D printer settings optimized for torsional strength. It is the main objective of this study to determine which settings contribute 
most to torsional strength by specifically altering specific FFF 3D printer settings of a test part, testing that test part in a custom testing machine, and comparing the results to parts printed with unmanipulated settings and other series of settings. Additional objectives include creating FFF 3D printer settings optimized for torsional strength per US dollar and torsional strength per minutes spent printing.

\section{Definitions}

3D Printing: An additive manufacturing process where digital designs made in CAD software are constructed in a layer-by-layer manner, allowing for geometrically complex models to be reproduced quickly. However, due to the Square-Cube Law, scaling up the production of 3D prints quickly becomes infeasible as the volume of parts quickly outpaces their surface area.

Fused Filament Fabrication (FFF): A 3D printing technology common among hobbyists where molten plastic is extruded through a nozzle along a predetermined pattern on a single plane. This layer is then repeated until a complete model is formed. Deviations in the layer pattern allow for complex shapes to be formed.

Layer Adhesion: In reference to the weld strength between different layers of an FFF 3D print.

Anisotropic: Indicates nonuniformity along a part or material such that testing along different axes yields different results. For example, the layer-by-layer process of 3D printing means it is extremely resistant to decompression along the $\mathrm{x}$ and $\mathrm{y}$ axes, but highly susceptible to decompression along the $\mathrm{z}$ axis.

Polylactic Acid (PLA): An extremely brittle biodegradable plastic that is widely available as filament for FFF 3D printing.

Computer-Aided Design (CAD): Parts are typically designed in this software. Add-ons within the program can expand it to encompass slicing, manufacturing, and simulation capabilities.

Tolerances: The margin of error between the design dimensions and the real dimensions of a part that must be accounted for during design (denoted by a $\pm x$ length). While certain manufacturing technologies can achieve low tolerances, the issue can never truly be eliminated.

Torsion/Torsional Strength: A twisting force exerted around the center axis of a part analogous to torque. Such strength refers to the maximum load a part can withstand when said load is applied torsionally.

Polar Moment of Inertia: Analogous to torsional strength except that it relies on specific assumptions like uniformity along all faces of the part ("Torsional Shear Stress Formula," 2020).

Shear Stress: Analogous to torque/torsion. When calculating this value, assumptions, like those of the polar moment of inertia, must be accounted for ("Torsion of Shafts," 2005).

Slicer/Slicer Profile: A program used to convert a 3D model into instructions for a 3D printer to follow. Profiles of this nature define specific settings and parameters that the program must incorporate into the model's instructions.

Infill Percentage: Defines the ratio of plastic to air within a 3D print. For example, $66 \%$ infill indicates a part filled with $34 \%$ air. Increasing this value is known to increase material usage and is believed to increase strength. 
Wall Line Count: Defines the number of walls or shells between the external environment and the infill. Increasing this value is known to increase material usage and is believed to increase strength.

Note that other unfamiliar terms may appear throughout this paper, but these will be defined in text instead of in Definitions because their appearance rate is too low to warrant a specific definition. Additionally, letter variables are used to explain specific concepts. Unless otherwise stated, these have no impact on any text outside of the variable's immediate vicinity.

\section{Literature Review}

Since superior technology is often available at the corporate and researcher level, there is a significant lack of research specifically on hobbyist FFF 3D printers and the properties of these manufactured parts. However, one can turn to studies that, despite not being aimed at FFF 3D printing, are still applicable to generating adequate hypotheses on the torsional properties of FFF 3D prints. For example, a study by the Journal of Aeronautical Scientists studied the torsional strength of a pure and unblemished plastic cylinder. After introducing various imperfections and thus anisotropicity, the researchers found that these imperfections often caused the cylinder to fail faster. Furthermore, these cylinders tended to fracture at these blemishes, signaling torsional stress targets the weakest point of a part rather than spreading uniformly (Lee, 2012). This draws parallels with the layer-by-layer manufacturing process of FFF 3D prints, where anisotropicity is consistently present.

Therefore, reducing anisotropicity immediately becomes an important objective for maximizing torsional strength in this study. While it can never be fully eliminated because of the aforementioned nature of FFF 3D printing, the degree of anisotropicity can be reduced by increasing precision since tolerances shrink. Researchers explored this reduction on a MakerBot 3D printer, where they altered different settings in an attempt to achieve the highest dimensional accuracy. The researchers concluded that the setting to have the greatest adverse impact on dimensional accuracy was infill percentage. At extreme percentages, infill would either bulge, increasing the overall dimensions of the printed part or not bond well to the walls of the part, allowing the walls to sag between the gaps in infill. However, researchers were also able to discover specific infill percentages that provided adequate support without bulge (Melenka, 2015). Unfortunately, further results were locked behind a paywall. However, gleaning the polarized nature of these failures, it is likely that an infill percentage near $50 \%$ would meet these conditions. Therefore, during testing, one variant infill percentage tested will be proximal to $50 \%(66 \%)$.

An alternative method to drawing on non-3D-printer-related studies could be to look towards the more common, but still rare, research surrounding tensile strength in FFF 3D prints. As opposed to torsional strength, tensile strength is where loads are applied perpendicularly to the base of the part. Therefore, loads are applied parallel to the central axis of the part instead of around this axis. In a study from the Departamento de Ingeniería Mecánica, researchers aimed to quantify the strength yield from increasing infill percentage. The study found that the tensile strength of pure ABS plastic differed by under 1\% from FFF 3D printed ABS with 100\% infill (Fernandez-Vicente, 2016).

However, a connection is still yet to be established between torsional and tensile strength. This connection was found by I-Kuang Fang and Jyh-Kun Shiah in an investigation into the "torsional strength and deformation for normal- [NSC] and high-strength concrete [HSC]." With HSC having a tensile strength gain of $35 \mathrm{MPa}$ compared to NSC, a similar positive gain in torsional strength was observed in HSC. Under similar conditions, comparing the tensile strengths of NSC and HSC served as a good indicator of the concrete possessing greater torsional strength (IKuang Fang, 2004).

Following these findings, it becomes reasonable to assume that slicer settings beneficial to FFF 3D print's tensile strength would be similarly beneficial to their torsional strength. In a posted test, YouTuber CNC Kitchen tested various samples and determined infill percentage and wall line count to have the greatest effect on prints' tensile strength ("INFILL pattern and SHELLS," 2018). Thus, these specific slicer settings were tested. 
Given the above literature, it is justifiable to ask the question: How can slicer profiles be best optimized for torsional strength within FFF 3D prints? The described contact is predicted to be far more prevalent during the manipulation of infill settings than any other slicer setting.

\section{Hypotheses}

1. Increasing infill percentage will increase time lasted and torsional strength.

- Following the formula for the polar moment of inertia, an increase in base surface area directly correlates to torsional strength. By increasing the plastic to air ratio in parts, plastic-to-plastic contact between layers is increased, causing an increase in torsional strength.

2. Increasing wall line count will also increase time lasted and torsional strength.

- Similarly, additional shells increase the contact between layers in an FFF 3D print. Per the formula for the polar moment of inertia, an increase in torsional strength should be observed.

3. Infill percentage will have a greater impact on time lasted and torsional strength than wall line count.

- Since infill percentage has a greater effect on each layer's surface area than wall line count, it is predicted to have a greater effect on torsional strength as a whole.

\section{Methods}

Within this study, three custom parts and one custom assembly were utilized, all of which can be assigned to the categories Testing Machine or Test Parts. All designs were created and sliced in software readily available to the author: SOLIDWORKS 2018 Education Edition and Ultimaker Cura 4.3.0 respectively.

\section{Testing Machine}

The testing machine was designed as a gearbox to significantly increase the torque of a NeveRest Classic 40 Gearmotor. Aluminum bars joined with plastic brackets were chosen as the primary build system for their availability and rigidity. Additionally, $1 / 8$ in acrylic plates help secure the frame together and act as mounting points for the bushings and other rotating components. 


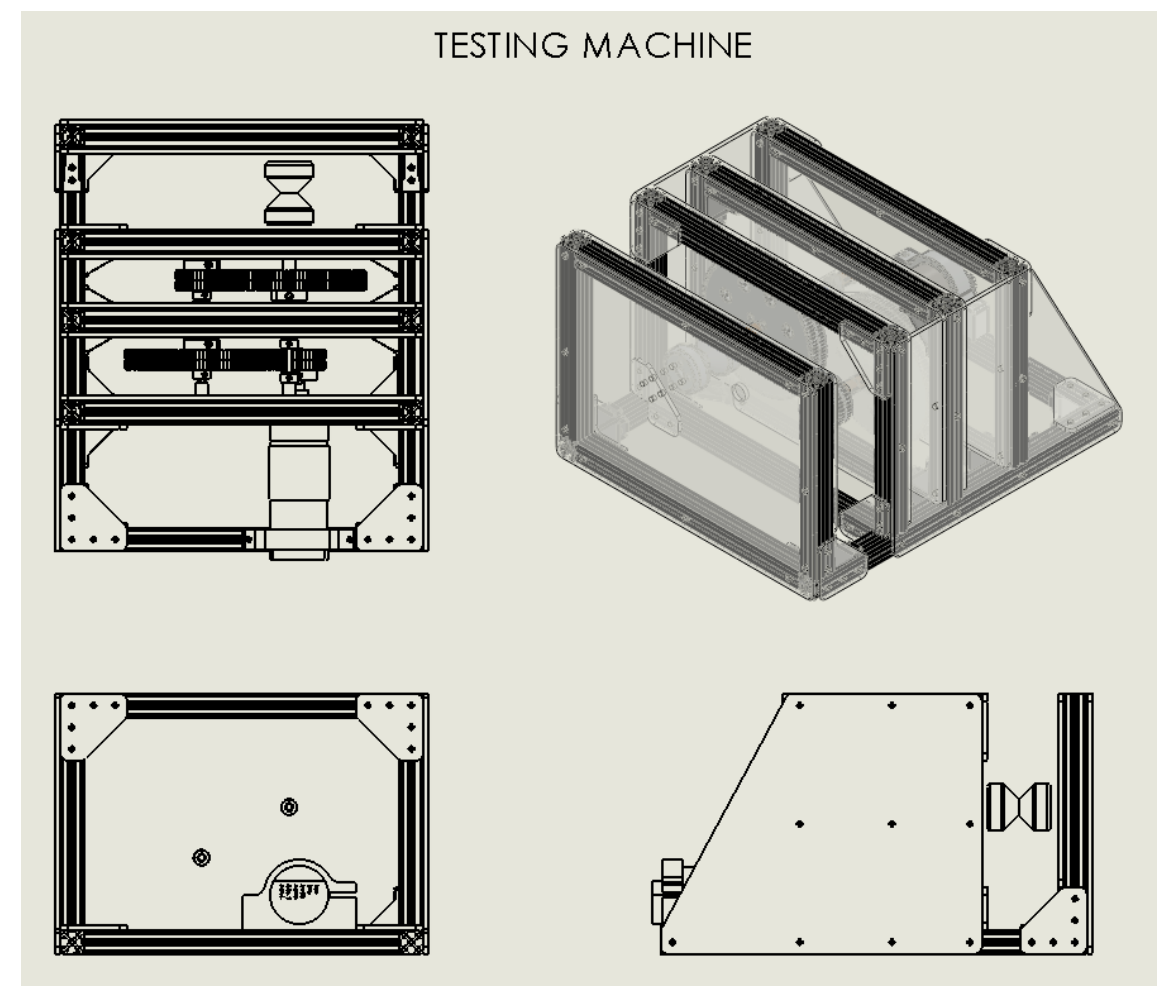

Figure 1. Testing Machine. Engineering drawings of testing machine from different views and an isometric render.

Using 2 sets of TETRIX® MAX 40 Tooth Gears to TETRIX® MAX 120 Tooth Gears, a 9:1 gear ratio was applied to the NeveRest 40 Classic Gearmotor's input shaft. This high gear reduction ensured that the output shaft, and subsequently the TETRIX® MAX Axle Hub which test parts were mounted to, applied the greatest possible torque. These gears were secured to axles with the aforementioned set-screw-based hubs. In turn, these axles rotate on bushings to reduce friction and conserve torque. Additionally, bearings are unable to take forces running parallel to the length of the axle they support, and the specific loads the testing machine would experience were uncertain.

The testing machine utilizes a relay switch to regulate the flow of electricity between the power supply and the motor, allowing for unidirectional movement. The relay detects a signal from an ELEGOO UNO R3 Board ATmega328P ATMEGA16U2, an ARDUINO UNO R3 [A000066] clone. From there, the code begins spinning the motor clockwise, turning the output shaft and applying a clockwise torque to the test part.

\section{Test Part-Model A}

Model A was designed with an hourglass-shaped body. Since torsion is applied circularly, Model A's circular profile prevents the concentration of torsional stress at specific points. 


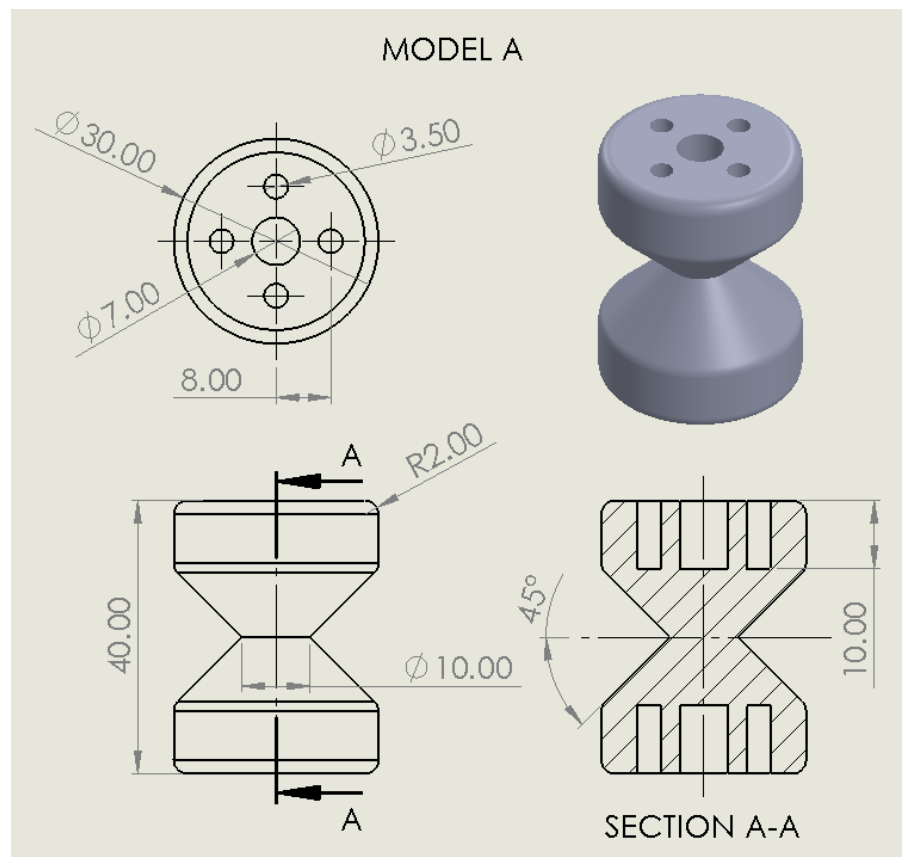

Figure 2. Model A. Engineering drawings of Model A from different views and an isometric render. Note the indicated lines of symmetry when determining additional nominal dimensions. All fillets have a $2.00 \mathrm{~mm}$ radius.

The hourglass design focuses all torsion along a single plane: the midplane of Model A. By consistently applying the same torque to the same area of each part during each test, variability between each test can be reduced. The $8.00 \mathrm{~mm}$ hole pattern on the bottom and top faces of Model A are designed to be tapped and accept 6-32 screws of up to 0.5 in in length. While these lengths do exceed the specified $10.00 \mathrm{~mm}$, these lengths are necessary to secure the part to the $6.70 \mathrm{~mm}$ thick TETRIX® MAX Axle Hub and acrylic plate setup during testing. The center $7.00 \mathrm{~mm}$ hole allows for excess axle length to run through the part, making flush mounting easier.

\section{Test Part-Model B}

Model B possesses much of the same qualities as Model A, including the same mounting pattern and nominal cylindrical dimensions. However, the difference lies in the diameter of the plane of the model where torque is focused. Whereas Model A had a minimum diameter of $10.00 \mathrm{~mm}$, Model B has a minimum diameter of $15.00 \mathrm{~mm}$. Increasing this diameter allows for a greater range in data collected and offers greater support that the results of this study are applicable to parts of all thicknesses. 


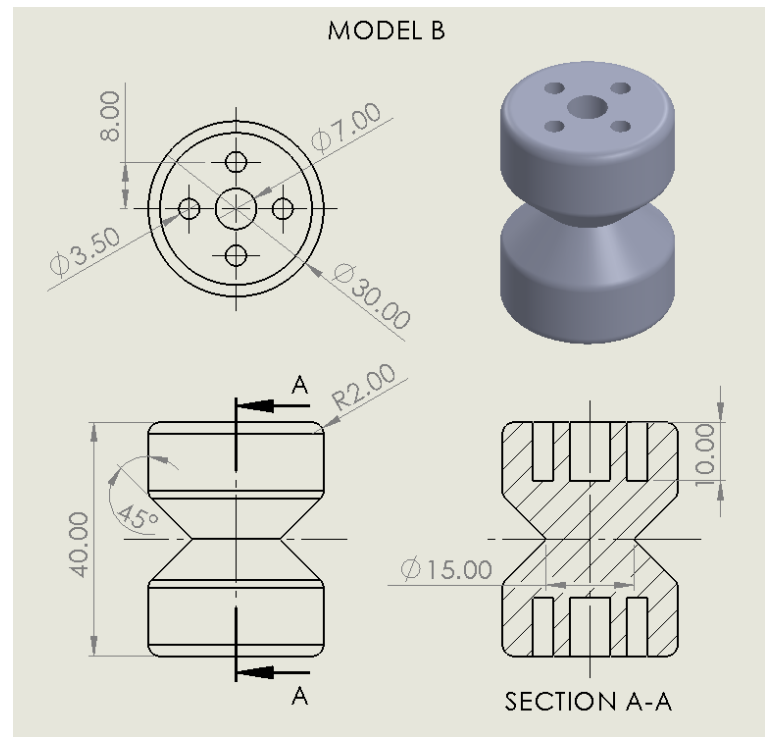

Figure 3. Model B. Engineering drawings of Model B from different views and an isometric render. Note the indicated lines of symmetry when determining additional nominal dimensions. All fillets have a 2.00 mm radius.

\section{Test Part-Model C}

Model $\mathrm{C}$ again retains the same nominal cylindrical dimensions and mounting pattern of both Models A and B. However, it lacks the hourglass shape and is instead a pure cylinder, distinguishing it from previous models. This encourages more normal torsion forces across the length of the part rather than at a specific point. Results from Model $\mathrm{C}$ were expected to be more variable than results from either Models A or B because of this induced normality.

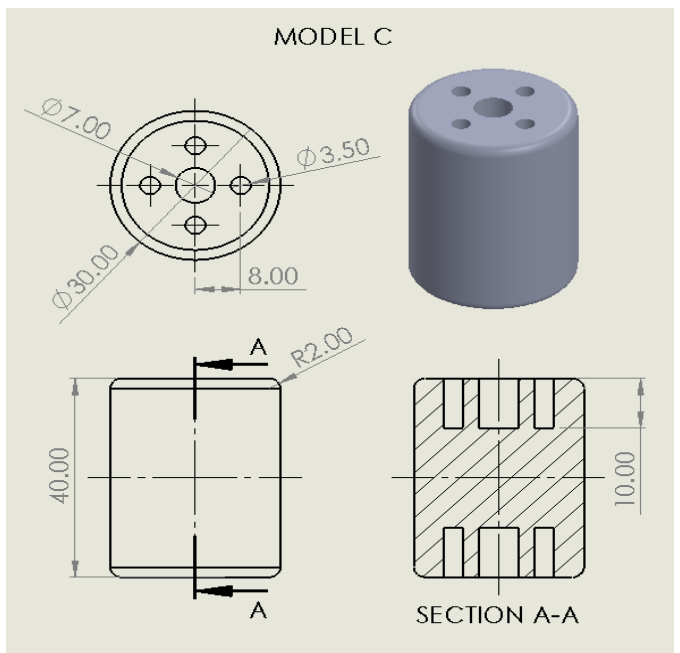

Figure 4. Model C. Engineering drawings of Model C from different views and an isometric render. Note the indicated lines of symmetry when determining additional nominal dimensions. All fillets have a $2.00 \mathrm{~mm}$ radius.

\section{Filament Material}

Every part was 3D printed out of Amazon Basics Orange PLA. PLA was chosen because of its brittle nature, which ensures the part will snap rather than slowly deform. Since observing deformation is far more subjective than observing a snap, brittle FFF 3D prints are key to more objective results. 


\section{Experimental Design}

The experimental design focused heavily on redundancy, with each settings and model combination being tested twice. With five possible settings combinations per model spread across three different models, this amounts to a sample size of 30 test parts. Each part is denoted as Part X\#, with X referring to the model designation and \# referring to a number from one to ten denoting the specific set of settings listed below. Each part is loaded into the testing machine. Once the part is inserted, the ground wire is unplugged as the code is uploaded. This prevents the code from immediately starting. Then, the timer is started as the ground wire is inserted into the appropriate pin. The timer is stopped as soon as the part is observed to break and this time is recorded. This procedure is repeated for all 30 parts within the sample.

\begin{tabular}{|c|c|c|}
\hline Part No. & Infill Percentage (\%) & Wall Line Count \\
\hline Part X1 & 33 & 3 \\
\hline Part X2 & 33 & 3 \\
\hline Part X3 & 66 & 3 \\
\hline Part X4 & 66 & 3 \\
\hline Part X5 & 100 & 3 \\
\hline Part X6 & 100 & 3 \\
\hline Part X7 & 66 & 2 \\
\hline Part X8 & 66 & 2 \\
\hline Part X9 & 66 & 4 \\
\hline Part X10 & 66 & 4 \\
\hline
\end{tabular}

Figure 5. Manipulated Settings. Table showing the part identification assigned to each slicer profile variant. Note that the control slicer profile with median settings is applied to Parts X3 and X4.

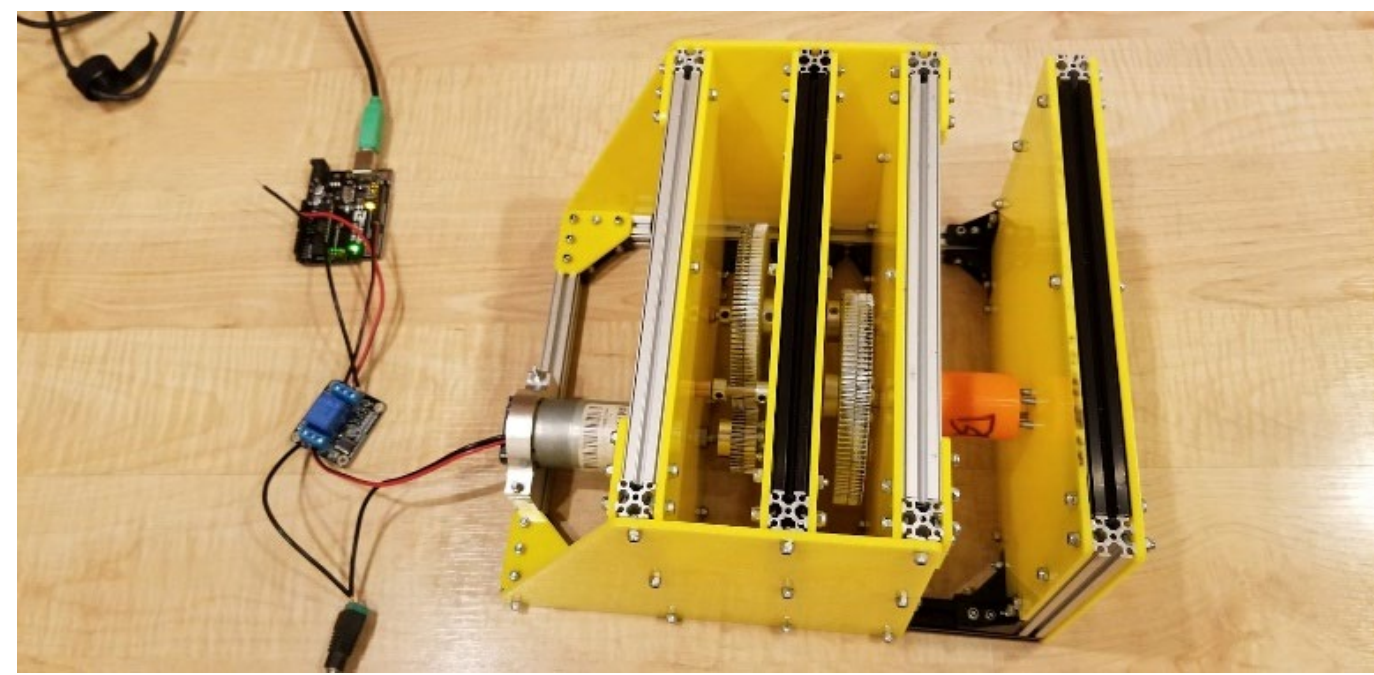

Figure 6. Testing Machine and Electronics. Picture of the testing machine with Part B1 loaded. Note the unplugged black ground wire in the top left corner. 


\section{Results}

Margin of Error:

A margin of error of $\pm 0.26 \mathrm{~s}$ was established by measuring the author's reaction time over five trials and averaging the result using the online software Human Benchmark.

Parts A1 - A10

\section{Findings}

Table 1. Infill percentage tests including control tests (Parts A3 and A4)

\begin{tabular}{|c|c|c|c|}
\hline Part No. & Infill Percentage (\%) & Wall Line Count & Time Lasted (s) \\
\hline Part A1 & 33 & 3 & 0.35 \\
\hline Part A2 & 33 & 3 & 0.92 \\
\hline Part A3 & 66 & 3 & 1.58 \\
\hline Part A4 & 66 & 3 & 0.98 \\
\hline Part A5 & 100 & 3 & 2.83 \\
\hline Part A6 & 100 & 3 & 1.15 \\
\hline
\end{tabular}

Table 2. Wall line count tests including control tests (Parts A3 and A4)

\begin{tabular}{|c|c|c|c|}
\hline Part No. & Infill Percentage (\%) & Wall Line Count & Time Lasted (s) \\
\hline Part A7 & 66 & 2 & 1.46 \\
\hline Part A8 & 66 & 2 & 0.98 \\
\hline Part A3 & 66 & 3 & 1.58 \\
\hline Part A4 & 66 & 3 & 0.98 \\
\hline Part A9 & 66 & 4 & 1.38 \\
\hline Part A10 & 66 & 4 & 0.90 \\
\hline
\end{tabular}

Table 3. Additional statistics on all parts

\begin{tabular}{|c|c|c|c|c|}
\hline Part No. & Filament Used $(\mathrm{g})$ & Cost (USD) & Print Time (min) & Time Lasted (s) \\
\hline Part A1 & 16 & 0.32 & 99 & 0.35 \\
\hline Part A2 & 16 & 0.32 & 99 & 1.58 \\
\hline Part A3 & 23 & 0.46 & 132 & 0.98 \\
\hline Part A4 & 23 & 0.46 & 132 & 2.83 \\
\hline Part A5 & 29 & 0.58 & 161 & 1.15 \\
\hline Part A6 & 29 & 0.58 & 161 & 1.46 \\
\hline Part A7 & 22 & 0.44 & 125 & 0.98 \\
\hline Part A8 & 22 & 0.44 & 125 & 1.38 \\
\hline Part A9 & 24 & 0.48 & 140 & 0.90 \\
\hline Part A10 & 24 & 0.48 & 140 & \\
\hline
\end{tabular}




\section{Infill Percentage Scatterplots}

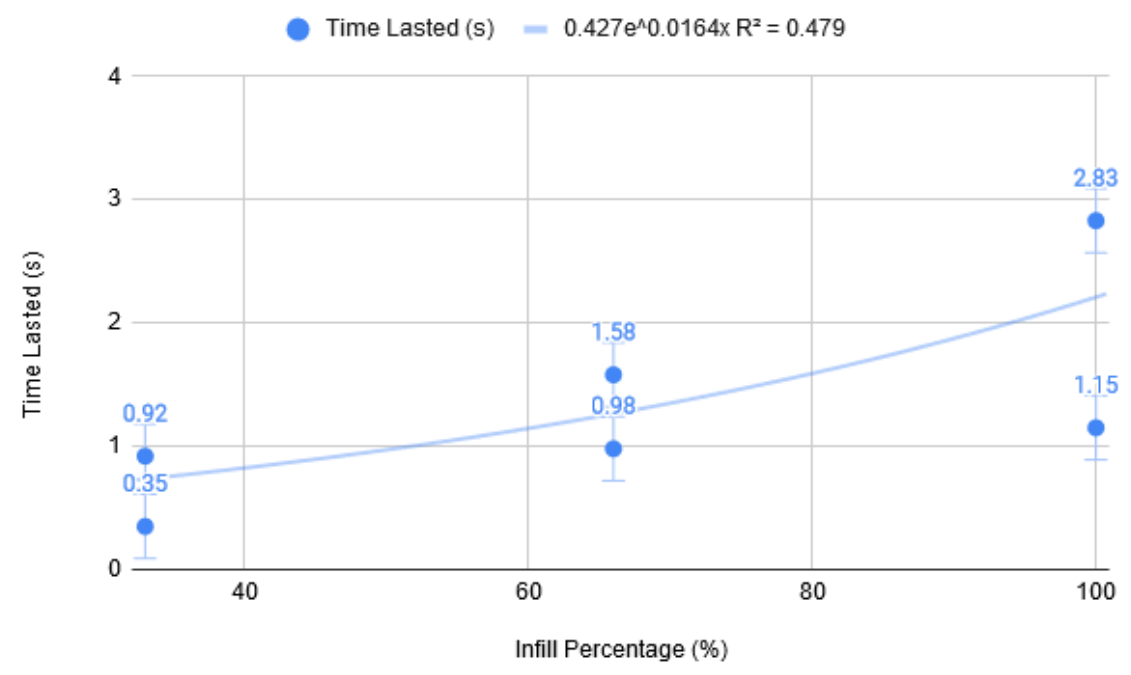

Figure 7. Infill Percentage vs. Time Lasted.

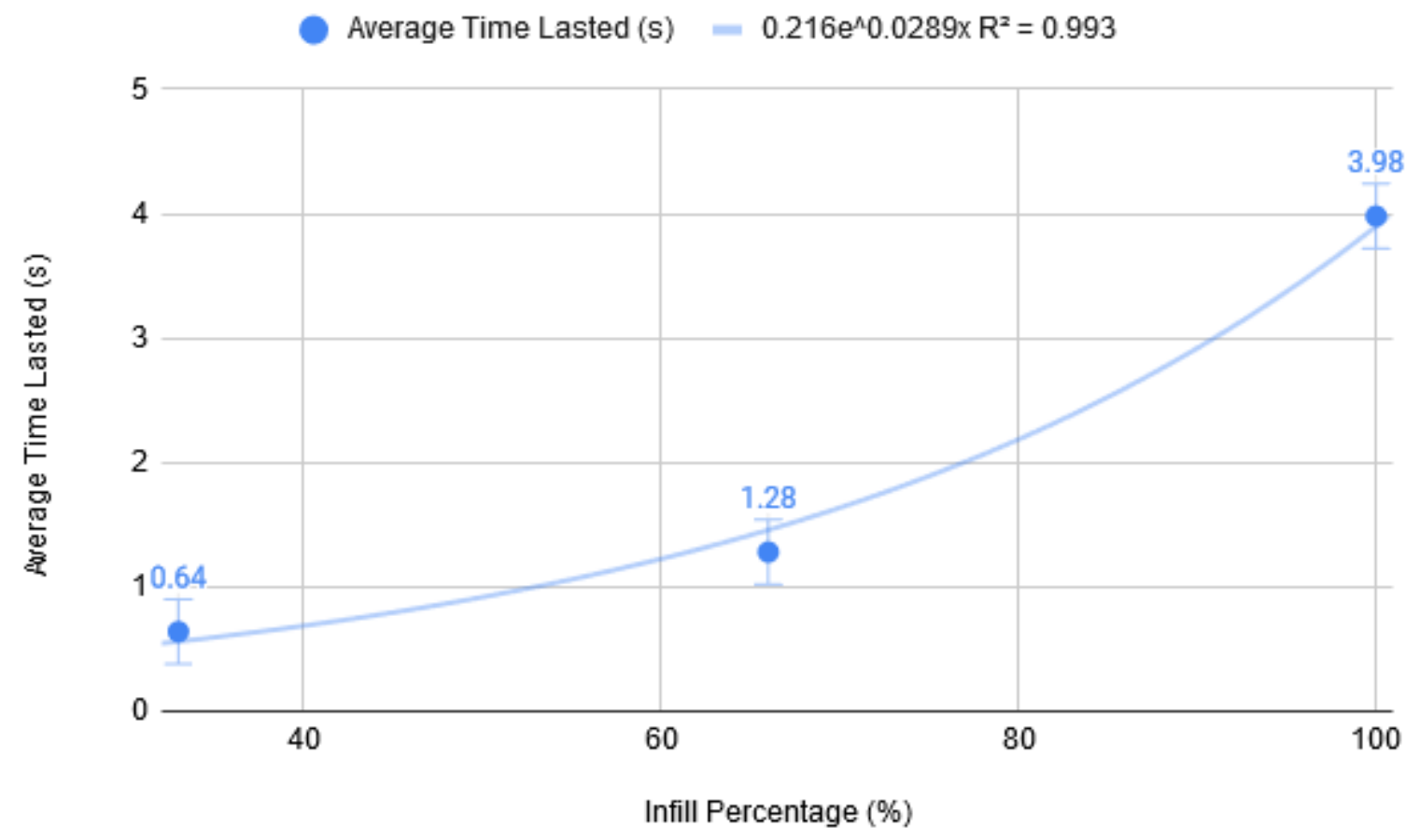

Figure 8. Infill Percentage vs. Average Time Lasted. 


\section{Wall Line Count Scatterplots}

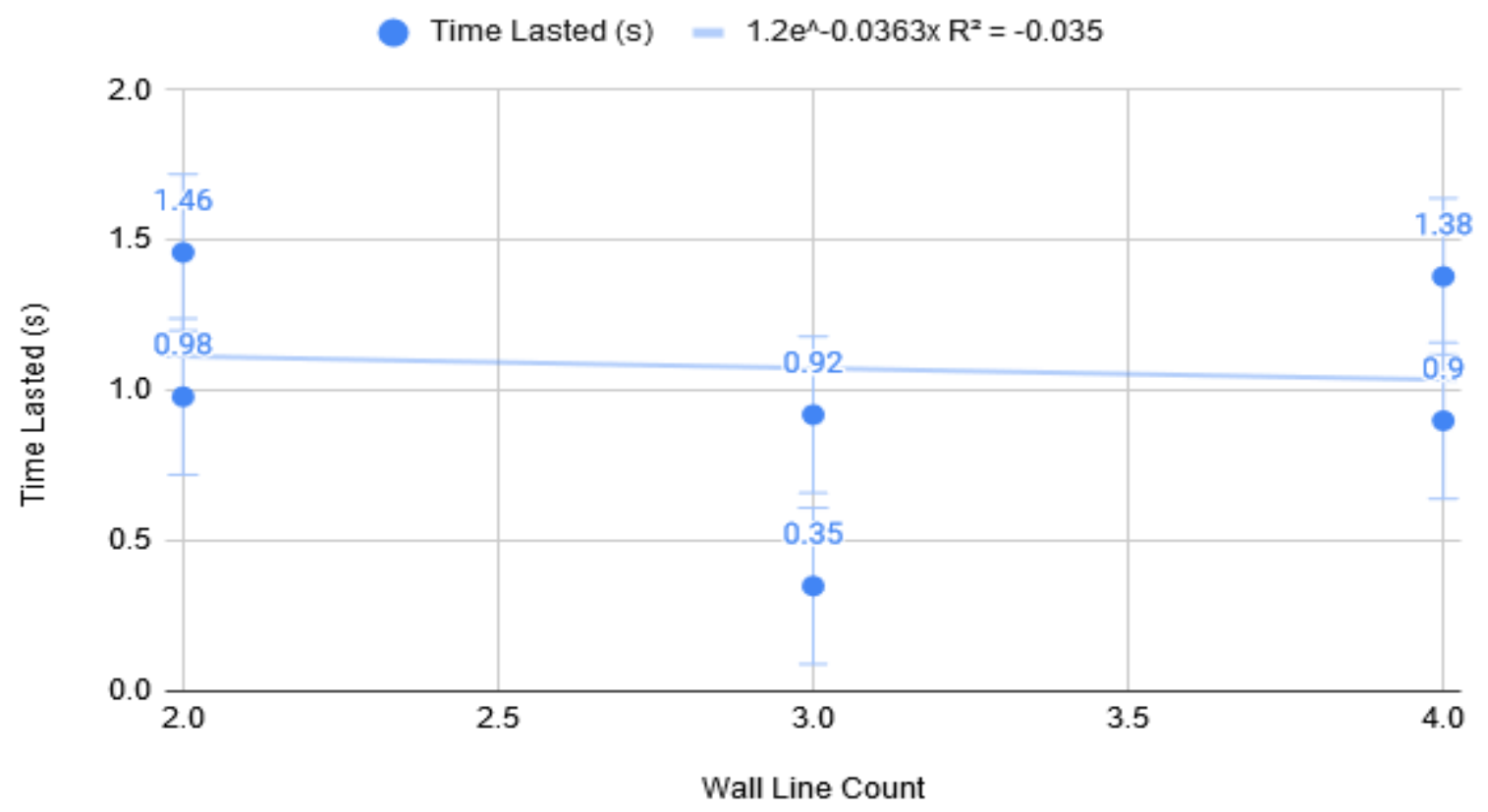

Figure 9. Wall Line Count vs. Time Lasted.

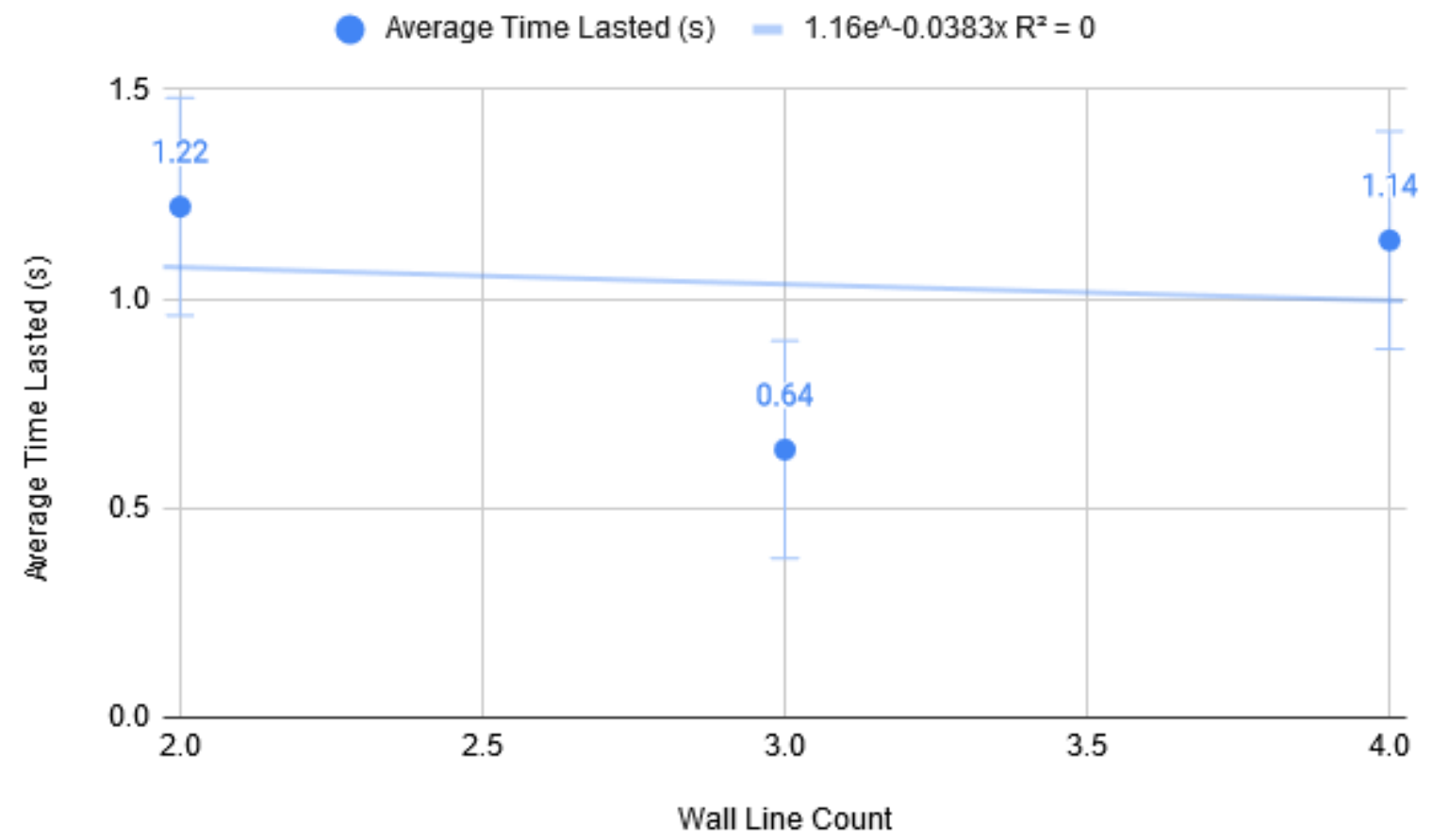

Figure 10. Wall Line Count vs. Average Time Lasted. 


\section{Additional Charts}

4

3

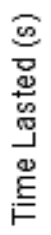

2

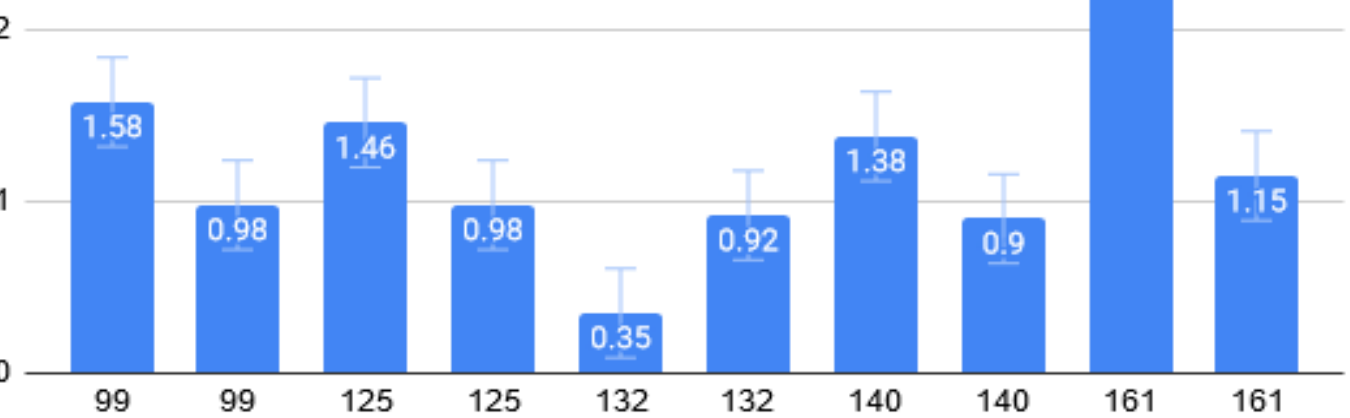

Print Time (min)

Figure 11. Print Time vs. Time Lasted (Column Chart).

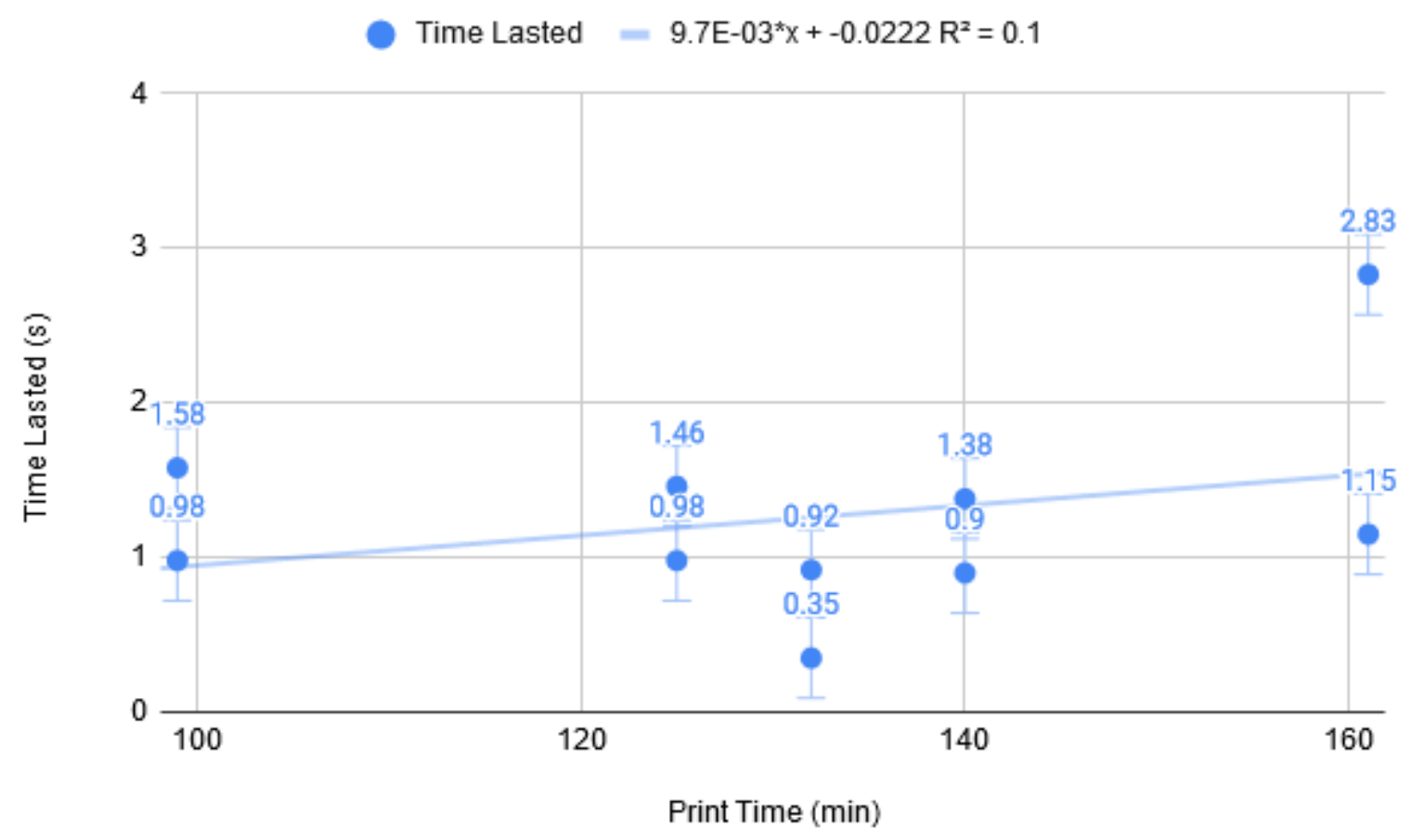

Figure 12. Print Time vs. Time Lasted (Scatterplot). 
In the tables and charts above, Time Lasted is synonymous with torsional strength. This is because Time Lasted refers to the time each part lasted in the testing machine before snapping. Additionally, note that both measurements of Filament Used and Cost are slicer-calculated estimates, and thus may vary from real world results. Real world costs were unobtainable because parts with different slicer profiles were printed simultaneously (Parts A8 and A9) due to time constraints. Print Time was similarly calculated within the slicer and excludes heating times since they vary from printer to printer.

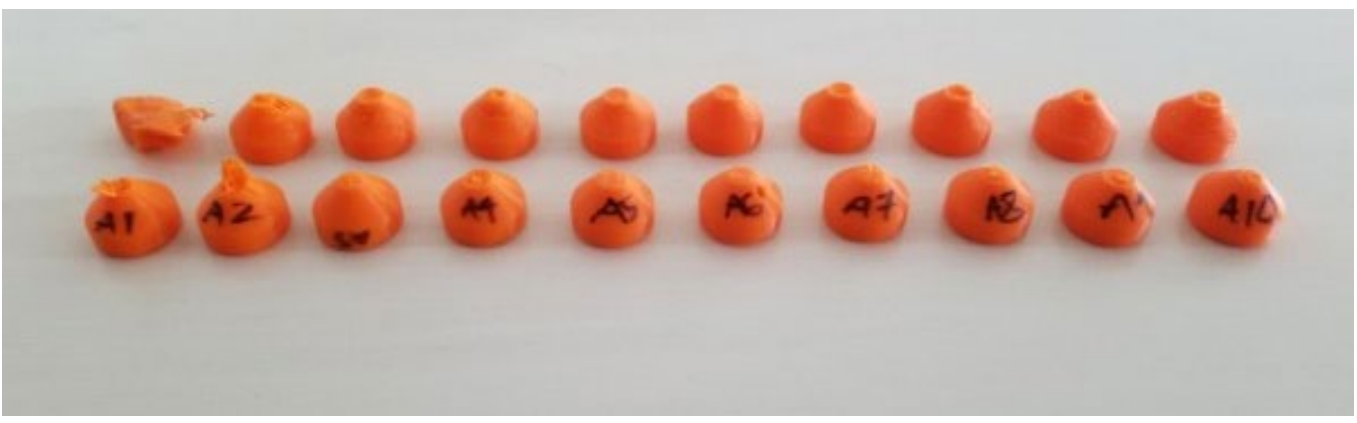

Figure 13. Parts A1 - A10. Picture of Parts A1 - A10 after testing. Note that the damage to the upper half of Part A1 pictured above was not sustained during testing. Rather, the part became difficult to remove from the testing machine after testing, and was simply melted and pried off. Therefore, no impact was made on RESULTS.

\section{Parts B1 - B10 and C1 - C10}

\section{Findings}

The testing machine was unable to snap Parts B1 - B10 and $\mathrm{C} 1-\mathrm{C} 10$ because of a set screw failure on the TETRIX® MAX Axle Hub. The set screw would dig a channel running widthwise along the flat of the TETRIX® MAX $4.7 \mathrm{~mm}$ Axle, enabling the set screw to rotate freely. Swapping the axle and hub with fresh replacements three times failed to yield different results. As such, Parts B1 - B10 and C1 - C10 were abandoned and no additional efforts were made to record their statistics.

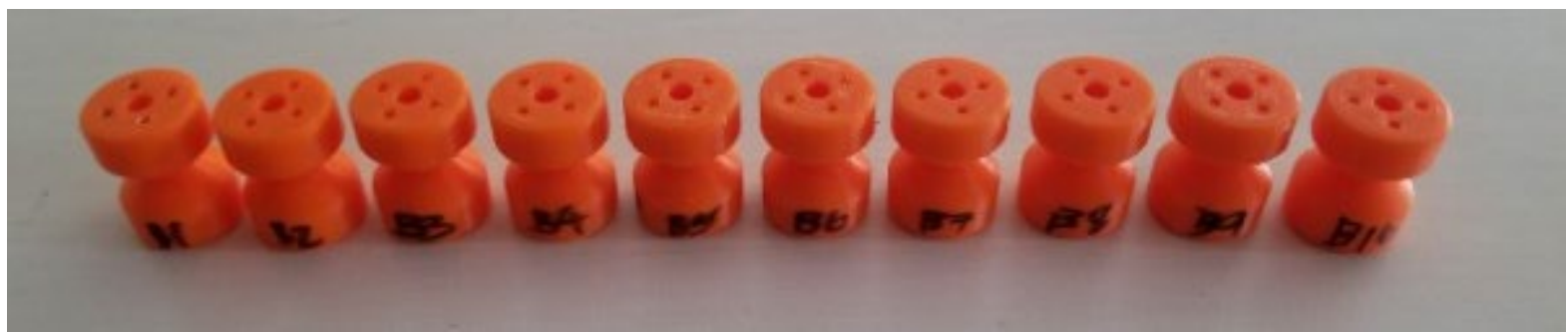

Figure 14. Parts B1 - B10. Picture of Parts B1 - B10 after testing.

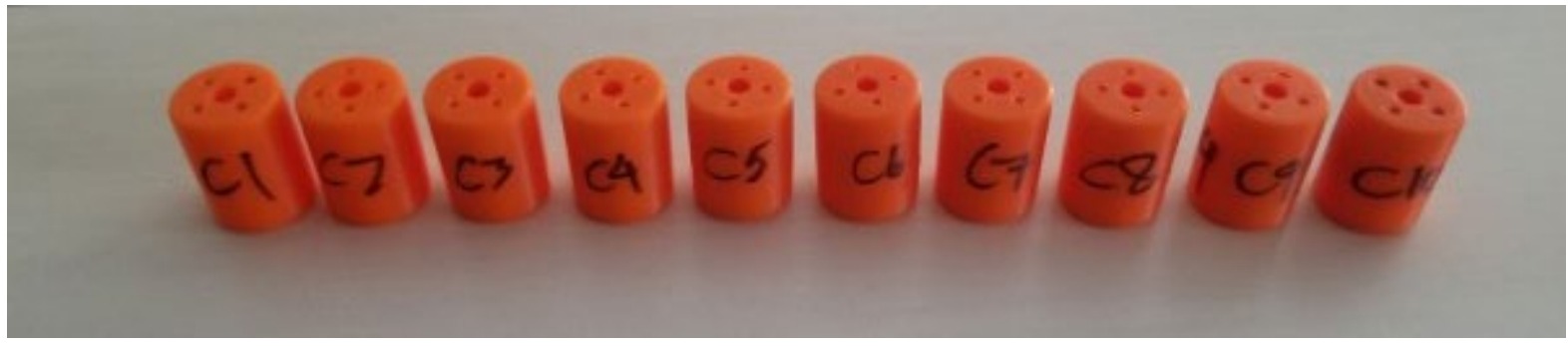

Figure 15. Parts $\mathrm{C} 1-\mathrm{C} 10$. Picture of Parts $\mathrm{C} 1-\mathrm{C} 10$ after testing. 


\section{Data Analysis}

\section{Infill Percentage}

As recorded in Figure 7, a clear positive correlation $(\mathrm{R}=0.692)$ was present between infill percentage and time lasted; an average increase in infill percentage tended to yield an overall increase in time lasted/torsional strength. Indicated by the trendline below, a 33\% increase in infill percentage yields approximately 0.67 additional seconds of time lasted. This linear simplification is valid because the derivative of the trendline from $33 \%$ to $100 \%$ varies minimally across the shown portion of the exponential plot. Exponential trendlines were used simply because the polar moment of inertia is an exponential function.

In Figure 8, the exponential nature of the graph becomes more apparent as the "cancelling effect" of two data points with the same infill percentage is replaced with the average time lasted. The stark difference in concavity comes as a result of the coefficient of $\mathrm{x}$ increasing by a factor of $75 \%$, again aided by the decrease in data points. While conclusions on a concrete mathematical relationship between infill percentage and average time lasted are inappropriate, conclusions can be made on the nature of their statistical relationship: an extremely strong positive correlation $(\mathrm{R}=0.996)$. The statistically significant positive correlation confirms Hypothesis 1 : increasing infill percentage would yield an increase in (average) time lasted and subsequently torsional strength.

\section{Wall Line Count}

At a glance, Figure 9 does not appear to show any significant correlation between wall line count and time lasted/torsional strength. Figure 10 supports this hunch: wall line count and time lasted appear to have a nearly horizontal trendline along the pictured interval. However, this evidence is shaky at best. $\mathrm{R}=0.187$, indicating a data set with an extremely high variability and therefore a lack of correlation between wall line count and time lasted.

Averaging both trials of wall line count, unlike with infill percentage, yields not a stronger correlation but no correlation whatsoever between wall line count and average time lasted because $\mathrm{R}=0$. Therefore, the trendlines in both Figure 9 and Figure 10 are irrelevant because their respective values of R are too low to be considered statistically significant. Additionally, the plotted trendlines can be considered further irrelevant because of their low $\mathrm{R}^{2}$ value, indicating a low adherence to the trendline.

\section{Miscellaneous Analysis}

As shown in Figure 11, the longest time lasted observed during testing was $2.83 \mathrm{~s}$. Even including the margin of error, $2.83 \mathrm{~s}$ still remains the longest time lasted by nearly a full second. However, this maximum does not aid in illustrating or clarifying a trend or correlation between time lasted and print time, which is further confirmed by Figure 12.

This scatterplot showcases a linear trend approaching a horizontal slope. Therefore, print time and time lasted do not have a correlation. Further evidence comes from $R=0.316$, which is too low to be considered statistically significant.

\begin{tabular}{|c|c|c|}
\hline Part No. & Print Time $(\min )$ & Cost (USD) \\
\hline Parts A1/A2 & 99 & 0.32 \\
\hline Parts A7/A8 & 125 & 0.44 \\
\hline Parts A3/A4 & 132 & 0.46 \\
\hline Parts A9/A10 & 140 & 0.48 \\
\hline Parts A5/A6 & 161 & 0.58 \\
\hline
\end{tabular}


Figure 16. Print Time Versus Cost. Table showing the time and cost in USD it took to print parts using the same slicing profile variant. Note that print time and cost are proportional, meaning the lack of a correlation between print time and time lasted applies to cost. Therefore, there is no correlation between cost and time lasted.

\section{Discussion}

\section{Hypotheses Revisited}

1. Infill percentage was proven to increase (average) time lasted and thus torsional strength.

- This justification held true. The increase in surface area afforded by a greater infill percentage significantly increased torsional strength.

2. Wall line count was proven to have no correlation with (average) time lasted, disproving this hypothesis.

- Reevaluating the justification behind this hypothesis, one glaring issue stands out: the additional surface area provided by increasing the number of shells is almost negligible compared to the that gained by increasing the infill percentage. While a slight positive correlation should be observable between wall line count and time lasted, this likely passed by unnoticed because of the margin of error of $\pm 0.26 \mathrm{~s}$.

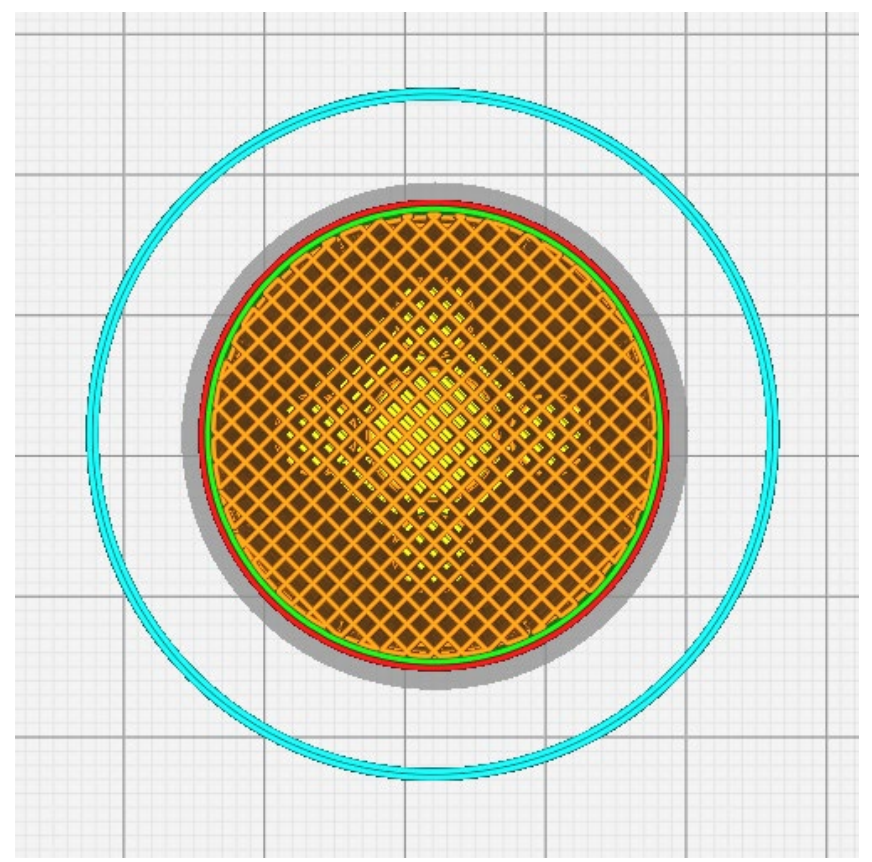

Figure 17. Model A Cross-Section. Picture of a cross-section of Model A sliced along a plane parallel to the model's top face from within Ultimaker Cura 4.3.0. Note that the surface area offered by the shells (shown in red and green) is next to nothing compared to that offered by the infill (shown in orange).

3. Infill percentage was therefore proven to have a greater impact on torsional strength/(average) time lasted than wall line count.

- Naturally, since Hypothesis $\mathbf{1}$ was proven while Hypothesis $\mathbf{2}$ was disproven, the described setting of $\mathbf{H y -}$ pothesis 1, infill percentage, is torsionally stronger than that described in Hypothesis 2, wall line count. 


\section{Limitations and Future Directions}

While unexpected, Parts B1 - B10 and C1 - C10 proved to be too torsionally stiff for the testing machine to snap. The failure point proved to be the axle and set screw system. A different build system would likely be able to handle more torque and potentially snap Parts B1 - B10 and C1 - C10. For example, clamping hubs sold by other companies can replace a set screw system, allowing for more security than the PITSCO TETRIX build system used during this experiment. Alternatively, one could swap the axle being used (a D-shaft design) for one with a hexagonal shaft, making set screws unnecessary altogether. A stronger axle retention system would allow for a greater number of test parts to be broken, increasing the overall sample size and accuracy of the study.

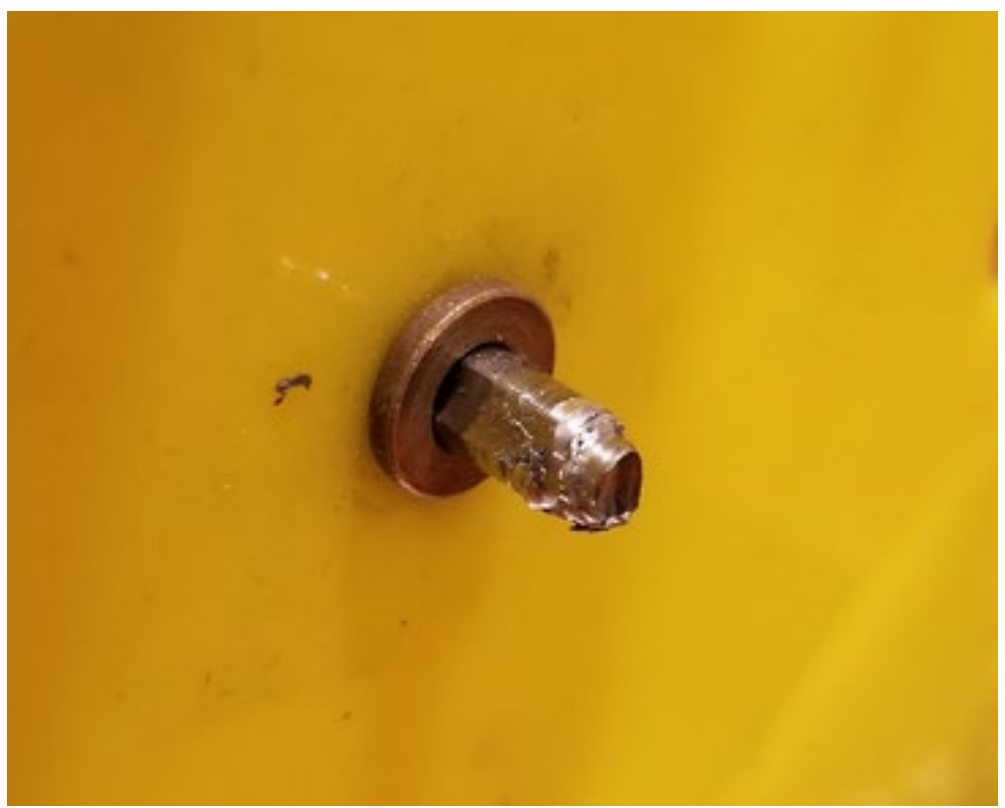

Figure 18. Stripped Axle. Picture of one of three stripped axles incurred while testing Parts B1 - B10 and C1 - C10.

Since more accurate methods of time-keeping were initially unavailable, there is an inherent inaccuracy with timing: the $\pm 0.26 \mathrm{~s}$ margin of error during data collection. Therefore, should this experiment be replicated, a need arises for a way to accurately measure the time between the motor starting and the test part snapping. This could be accomplished with an external encoder that reads the position of the test part. When the test part snaps, it will begin to spin, which the encoder can precisely detect and precisely time in relation to the start of the motor. This could help illustrate more accurate trendlines with greater values for $\mathrm{R}^{2}$.

Due to the nature of FFF 3D printing, two parts, even when printed with the exact same slicer profiles under the exact same conditions, will likely have obvious differences in surface finish and minute differences in strength (as proven by the different values of time lasted between Parts A1 and A2, A3 and A4, etc.). Unlike the aforementioned limitations, this is simply an issue that can be mitigated, although not completely solved, by tuning one's slicer profiles.

However, the greatest issue with this study is the low sample size. While a strong correlation was shown between infill percentage and time lasted/torsional strength, any conclusions made must be tentative since only six samples were tested. With greater sample sizes and tests on a greater variety of models, a more revealing picture can be painted on the relationship infill percentage and wall line count have with torsional strength. 


\section{Conclusion}

While only a tentative relationship between infill percentage and torsional strength can be proposed, the evidence found thus far does overwhelmingly suggest such a relationship exists. While the author is confident in recommending infill percentage as a must when 3D printing hubs, pulleys, or torsionally demanding parts, further research must be conducted to determine the degree and precise correlation of their relationship.

\section{Acknowledgements}

Thank you to all the teachers at Santa Clara High School for teaching me the foundations of research and presentation during AP Seminar and AP Research. Additionally, thank you to the staff of the 49ers STEM Leadership Institute for supporting my project by manufacturing many of the custom components in my research. Thank you to Mrs. Flowers for helping me access the school laser cutter and for supporting me as my AP Research teacher.

\section{References}

CNC Kitchen. (2018). INFILL pattern and SHELLS - How to get the maximum STRENGTH out of your 3D prints? - YouTube. Retrieved May 15, 2020, from https://www.youtube.com/watch?v=AmEaNAwFSfI

Ender 3 3D Printer. (n.d.). Retrieved from https://www.creality3dofficial.com/collections/top-sell/products/officialcreality-ender-3-3d-printer

Evaluation of dimensional accuracy and material properties of the MakerBot 3D desktop printer. (n.d.). Retrieved from https://www.emerald.com/insight/content/doi/10.1108/RPJ-09-2013-0093/full/html

Fernandez-Vicente, M., Ferrandiz, S., Conejero, A., Andrzejewska, A., Fernandez-Vicente, M., Instituto de Diseño, . . Departamento de Ingeniería Mecánica. (2016, September 01). Effect of Infill Parameters on Tensile Mechanical Behavior in Desktop 3D Printing. Retrieved from https://www.liebertpub.com/doi/abs/10.1089/3dp.2015.0036

Global 3D printing market hits 500K units milestone. (n.d.). Retrieved from https://www.3ders.org/articles/20151125-global-3d-print-market-hits-500k-units-milestone.html

Human Benchmark. (n.d.). Retrieved July 29, 2020, from https://humanbenchmark.com/

I-Kuang Fang, J. S. (2004, May 01). Torsional Behavior of Normal- and High-Strength Concrete Beams. Retrieved from https://www.concrete.org/publications/internationalconcreteabstractsportal/m/details/id/13090

(n.d.). Retrieved from https://study.com/academy/lesson/torsional-shear-stress-formula.html\#transcriptHeader

Plastic Torsional Buckling Strength of Cylinders Including the Effects of Imperfections. (1957). Journal of the Aeronautical Sciences, 24(4), 241-248. doi:10.2514/8.3820

The State of 3D Printing 2019 Edition. (n.d.). Retrieved from https://cdn2.hubspot.net/hubfs/5154612/downloads/Sculpteo The State of 3D Printing 2019.pdf 
“Torsion of Shafts.” Engineering ToolBox, www.engineeringtoolbox.com/torsion-shafts-d 947.html

All models, assemblies, and engineering drawings were designed and screenshotted in SOLIDWORKS 2018 Education Edition and all slicer screenshots were taken in Ultimaker Cura 4.3.0. 